\title{
Intron Mutations Affect Splicing of Saccharomyces cerevisiae SUP53 Precursor tRNA
}

\author{
MARJORIE C. STROBEL ${ }^{1,2 \dagger}$ AND JOHN ABELSON ${ }^{1 *}$ \\ Division of Biology, 147-75, California Institute of Technology, Pasadena, California 91125, ${ }^{1}$ and Biology Department, \\ University of California, San Diego, La Jolla, California $92093^{2}$
}

Received 4 December 1985/Accepted 14 April 1986

\begin{abstract}
The Saccharomyces cerevisiae amber suppressor tRNA gene SUP53 (a tRNA ${ }^{\text {Leu }}$ allele) was used to investigate the role of intron structure and sequence on precursor tRNA splicing in vivo and in vitro. This gene encodes a pre-tRNA which contains a 32-base intervening sequence. Two types of SUP53 intron mutants were constructed: ones with an internal deletion of the natural SUP53 intron and ones with a novel intron. These mutant genes were transcribed in vitro, and the end-processed transcripts were analyzed for their ability to serve as substrates for the partially purified $S$. cerevisiae tRNA endonuclease and ligase. The in vitro phenotype of these mutant RNAs was correlated with the in vivo suppressor tRNA function of these SUP53 alleles after integration of the genes into the yeast genome. Analysis of these mutant pre-tRNAs, which exhibited no perturbation of the mature domain, clearly showed that intron structure and sequence can have profound effects on pre-tRNA splicing. All of the mutant RNAs, which were inefficiently spliced or unspliced, evidenced cleavage only at the $5^{\prime}$ splice junction. Base changes in the intron proximal to the $3^{\prime}$ splice junction could partially rescue the splicing defect. The implications of these data for tRNA endonuclease-substrate interactions are discussed.
\end{abstract}

Numerous studies have investigated yeast intragenic mutations which affect the production of a mature tRNA. These mutations map almost exclusively to the mature domain of the precursor tRNA $(2,3,11-13,16-18,21,35)$. In general, these mutations, which affect transcription, end-processing, and splicing of the precursor, perturb conserved base-pairing or alter conserved nucleotides in the mature domain. Notable by their paucity are mutations located in the intron. By genetic selection for an antisuppressor phenotype, only three intron mutants have been found.

In the Saccharomyces cerevisiae SUP4 $\mathrm{tRNA}^{\mathrm{Tyr}}$, a base change creates an RNA polymerase III transcription termination site in the intron (11). Willis et al. (35) found two intron alterations in the Schizosaccharomyces pombe sup 9 tRNA $^{\text {Ser: }}$ a base insertion which has little effect on RNA maturation or suppressor tRNA function and a base change near the 3' intron-exon junction. The latter mutation significantly decreases the efficiency of end-processing and splicing. In each case, the mutation(s) enhances the formation of alternate base-pairing between the mature domain and intron sequences, which perturbs the mature domain structure. Therefore, these intron mutants probably exert their effect on RNA processing by their alteration of total precursor tRNA conformation.

Mutations, selected for loss of suppressor tRNA function, which affect primarily splicing are found in the D stem-loop, the anticodon stem-loop, and the variable stem-loop (extra arm). As has been shown previously $(8,25,29)$, large intron alterations-base changes, insertions, and deletions-in $S$. cerevisiae tRNA precursors do not affect their absolute ability to be spliced. These observations have led to the following idea: if its sequence is not deleterious to the

\footnotetext{
* Corresponding author.

$\dagger$ Present address: LBI-Basic Research Program, National Cancer Institute-Frederick Cancer Research Facility, Frederick, MD 21701.
}

higher-order structure of the precursor, an intron plays no direct role in the maturation of a precursor tRNA. In short, the intron is passive in its own removal.

However, there is a single example of a nonspliced pretRNA which arises from a change in intron sequence. Raymond and Johnson (25) made a partial deletion of an $S$. cerevisiae tRNA ${ }_{3}^{\mathrm{Leu}}$ intron in vitro. In a cell extract of $S$. cerevisiae, this deletion mutant is efficiently transcribed and end-processed but is not spliced. The only obvious alterations in this mutant were the creation of an exceptionally small single-stranded region (two nucleotides) and an unconventional sequence (CC) at the $3^{\prime}$ intron-exon junction. These data suggest that the sequence or structure of the intron per se can be important in the splicing process.

To further investigate the role of the intron in splicing, mutants of the $S$. cerevisiae SUP53 (tRNA ${ }_{3}^{\mathrm{Leu}}$ ) gene were constructed. Two types of mutants were initially made: one carried an internal deletion of the natural SUP53 intron and one carried a novel intervening sequence (IVS). In each case, additional mutations were made in splice junctionproximal nucleotides. Each of the mutant genes was efficiently transcribed and its RNA product was efficiently end-processed in vitro with a yeast nuclear extract (YNE) preparation. Their splicing phenotype was determined in an in vitro assay with partially purified $S$. cerevisiae tRNA ligase and endonuclease. For several mutants, the identity of the products made in the presence of endonuclease and ligase was confirmed by RNA fingerprint analysis. Finally, the in vitro phenotype of several mutants was correlated with their tRNA suppressor function in vivo by integration of the mutant genes into the yeast genome.

These experiments show that certain intron-mutated precursors tRNAs are refractory to the tRNA-splicing endonuclease both in vitro and in vivo. Furthermore, secondary mutations at the $3^{\prime}$ intron-exon junction can partially rescue the nonspliced phenotype in vitro and in vivo. 


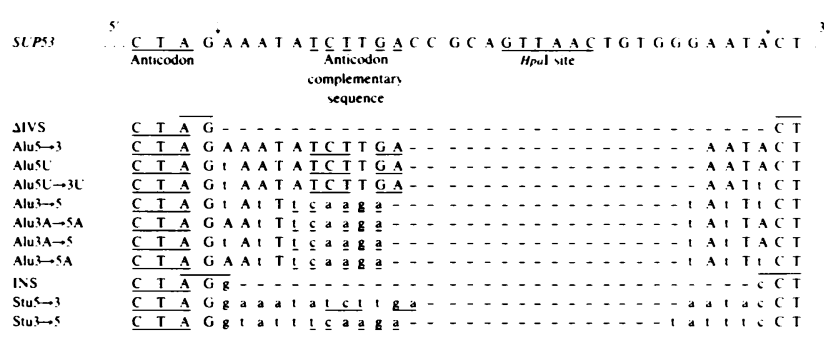

FIG. 1. Sequences of SUP53 intron mutants. The sequences of the SUP53 intron mutants examined are shown. Their constructed is described in detail in Materials and Methods. Arrows delimit the SUP53 32-base-pair intron. Underlined sequences show the anticodon (CTA) and the sequence, found in the SUP53 intron, which is complementary to the anticodon (TCTTGA). The dashed, underlined sequence is another intron sequence complementary to the anticodon (tcaaga). Lowercase letters emphasize differences in intron sequence from SUP53. Overlined sequences point out the unique, intragenic $A l u \mathrm{I}$ (AGCT) and StuI (AGgcCT) restriction enzymes sites created in the $\Delta$ IVS and INS mutants, respectively.

\section{MATERIALS AND METHODS}

Mutant construction. The strains, plasmids, and media used have been described previously (29). The mutants of the $S$. cerevisiae leucine-inserting, amber suppressor tRNA ${ }^{\text {SUPS3 }}$ investigated in this study are shown in Fig. 1. These mutants were made by oligonucleotide-directed mutagenesis or by the cloning of complementary oligonucleotides $(17,29)$. All mutants were initially constructed in M13mp10 and transferred to the yeast integration plasmid YIpLEU2 prior to their introduction into yeast cells (29).

In vitro transcription and splicing. YNE (4) was used to transcribe the SUP53-derived genes and to end-process the primary RNA transcripts $(17,29)$. The end-processed, [ $\alpha$ ${ }^{32}$ P]UTP-labeled transcripts were used in in vitro cutting and ligation assays $(7,23 \mathrm{a}, 29)$.

Yeast transformation and suppression testing. $S$. cerevisiae SL183-21C [ $\alpha$ met8-l(Am) tyr7-l(Am) trpl-1(Am) ade326(Am) ilvl-l(Am) leu2-l(Oc) lys2-l(Oc) can ${ }^{\mathrm{R}} 1-132(\mathrm{Am})$, kindly provided by $\mathrm{S}$. Liebman] was transformed with a linear DNA fragment containing both the SUP53 allele and the $L E U 2$ marker (29). Since these genes are closely linked on $S$. cerevisiae chromosome III $(1,5,15)$, integration of this fragment can both rescue the $\mathrm{Leu}^{-}$phenotype and introduce the SUP53 gene by homologous recombination (26).

$\mathrm{Leu}^{+}$transformants were secondarily screened for the function of the SUP53 allele by assaying their ability to

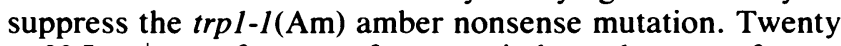
to $30 \mathrm{Leu}^{+}$transformants from two independent transformations with each SUP53 allele were examined by the method of Shaw and Olson (27) as previously described (29).

RNA analysis. The products of in vitro splicing assays were analyzed by RNA fingerprinting of RNase $T_{1}$ oligonucleotides by the methods of Volckaert et al. (34) and Volckaert and Fiers $(32,33)$. RNAs were precipitated with 20 to $40 \mu \mathrm{g}$ of carrier tRNA per sample and digested with RNase $T_{1}$ (2.5 to $5.0 \mathrm{U}$ in $10 \mathrm{mM}$ Tris chloride, $\mathrm{pH} 7.4,1 \mathrm{mM}$ EDTA; Calbiochem, La Jolla, Calif.) for 2 to $4 \mathrm{~h}$ at $37^{\circ} \mathrm{C}$. In the first dimension, samples were resolved by electrophoresis on cellulose-acetate strips equilibrated with $5 \%$ acetic acid-2 mM EDTA-5 M urea, pH 3.5, with pyridine. Electrophoresis was carried out with a high-voltage power supply (Savant Instruments, Hicksville, N.Y.). Samples were passively transferred essentially by the method of Southern (28) to polyethyleneimine-cellulose thin-layer plates (Cel 300 PEI; Brinkman Instruments, Westbury, N.Y.). Development of the second dimension was by homochromatography with $\mathrm{C}-10$ Homomix for 4 to $5 \mathrm{~h}$ at $65^{\circ} \mathrm{C}$. RNase $\mathrm{T}_{1}$ products were visualized by autoradiography on Kodak XAR5 X-ray film (Eastman Kodak, Rochester, N.Y.) with Cronex Lightning-Plus intensifying screens (I. E. duPont de Nemours, Inc., Wilmington, Del.) at $-70^{\circ} \mathrm{C}$ for 1 to 7 days.

Computer-determined RNA secondary structures. Secondary structures of SUP53 and mutant RNAs were determined by using the RNA sequence folding program of $\mathbf{M}$. Zuker, provided by the BIONET National Computer Resource for Molecular Biology. Structures, as drawn, were determined both as part of the total pre-tRNA and as subfragments spanning the anticodon stem-loop region (including IVS).

\section{RESULTS}

Mutant construction. The sequences of the intron mutants examined in this study are shown in Fig. 1. The mature tRNA domain in all of the mutants was unaltered SUP53.

The Alu series mutants were derived from the $\triangle I V S$ and Alu $5 \rightarrow 3$ mutants, which have been characterized previously (29). Briefly, the $\Delta$ IVS construction created a unique, intragenic $A l u I$ restriction enzyme site which precisely divided the tRNA gene into 5' and ' ${ }^{\prime}$ ' halves. Sets of complementary oligonucleotides were cloned into this site by blunt-end ligation. In the $5 \rightarrow 3$ orientation, the Alu mutants contained a 15-base intron which represented an internal deletion of the natural SUP53 IVS. The $3 \rightarrow 5$ orientation, the other ligation product, created a novel IVS. This novel intron contained a sequence complementary to the anticodon (Fig. 1). Potentially, the size and, certainly, the sequence of the splice junction-proximal loops were different from those found in SUP53 precursor.

The Stu family mutants were derived from the insert (INS) mutant characterized previously (29). By the insertion of two nucleotides, the INS mutant carried a unique StuI restriction enzyme site created from the $A l u I$ site found in $\Delta I V S$. Introduction of the complementary oligonucleotides into the site by blunt-end ligation created a 17-base intron. Again, the $5 \rightarrow 3$ mutant represented the SUP53 sequence, and the $3 \rightarrow 5$ mutant contained the novel intron.

In Fig. 1 the secondary nucleotide changes made by the cloning of complementary nucleotides or by oligonucleotidedirected mutagenesis are shown. The names of the mutants, derived from the original Alu and Stu constructions, reflect the secondary base changes.

In vitro splicing of Alu and Stu mutants. By using the YNE preparation (4), the initial Alu and Stu mutant genes were transcribed in vitro in the presence of $\left[\alpha-{ }^{32}\right.$ P]UTP. This extract contains the activities required to fully process the primary tRNA transcript $(17,29)$. All the mutant genes were efficiently transcribed and efficiently end-processed (data not shown). Since the activities which mature the $5^{\prime}$ and $3^{\prime}$ ends of a tRNA transcript are sensitive to the conformation of the mature domain, the efficient maturation of these transcripts suggests that these intron insertions are not significantly perturbing the appropriate precursor structure (29).

End-processed transcripts were recovered and used as substrates for the partially purified $S$. cerevisiae tRNA endonuclease ( $\sim 800$-fold purified; kindly provided by $C$. Peebles [22]) and ligase ( $\sim 400$-fold purified; kindly provided by E. Phizicky and C. Greer [7, 23a]) in the presence of ATP and $\mathrm{Mg}^{2+}$ (ligation conditions). Figure 2 displays the products of these assays. The transcripts were derived from a 
single transcription cocktail and, as such, were assumed to have virtually equal specific activity. Equal amounts of radioactivity of each transcript were used in each assay. Each RNA was tested in the presence of endonuclease alone and endonuclease plus ligase. Initially, the identity of bands was assigned by their size and their appearance and disappearance under appropriate conditions and confirmed by RNA fingerprinting (see Fig. 5 through 8).

Pre-tRNA ${ }^{S U P 53}$ and pre-tRNA ${ }^{\text {Alus } \rightarrow 3}$ produced two major products after endonuclease cleavage: $3^{\prime}$ and $5^{\prime}$ halfmolecules. With the addition of ligase, the half-molecules were depleted, and a dominant band of mature tRNA size appeared. SUP53 RNA also produced a 32-nucleotide-long IVS band. The smaller intron ( 15 bases) of Alu $5 \rightarrow 3$ was not retained on the gel.

The two Stu mutant RNAs generated undetectable amounts of 3 '-half and mature-size tRNA. The Alu3 $\rightarrow 5$ mutant generated a very small amount of these molecules. All three precursors produced some $5^{\prime}$ half-molecules and bands consistent in size with two-thirds-size molecules (IVS plus $3^{\prime}$ half) in the presence of endonuclease or endonuclease and ligase. Therefore, these mutant RNAs were refractory to complete endonuclease cutting.

In vitro splicing of Alu mutants with altered splice junctionproximal nucleotides. Two differences were immediately apparent between the introns of these precursors: the splice junction-proximal sequences and the total sequence (and structure) of the introns. The readily spliced Alu $5 \rightarrow 3$ mutant

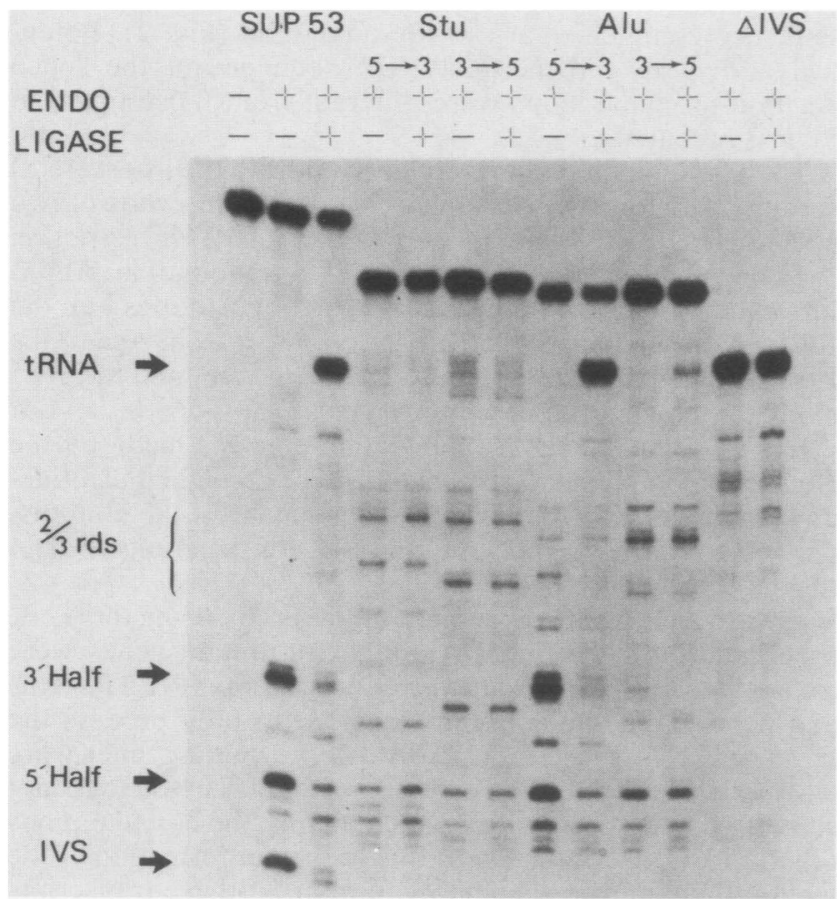

FIG. 2. In vitro splicing of SUP53 and intron mutants. Assays were performed as described in Materials and Methods. All endprocessed pre-tRNAs were derived from a single transcription cocktail, and equal Cerenkov counts were used in each cutting or splicing reaction mixture. Initially, the identity of products was based on their size and their appearance or disappearance under appropriate reaction conditions. The identity of bands, notably the two-thirds molecules, for selected mutants was confirmed by twodimensional fingerprint analysis of RNase $T_{1}$-digested RNAs. Endo, tRNA endonuclease.

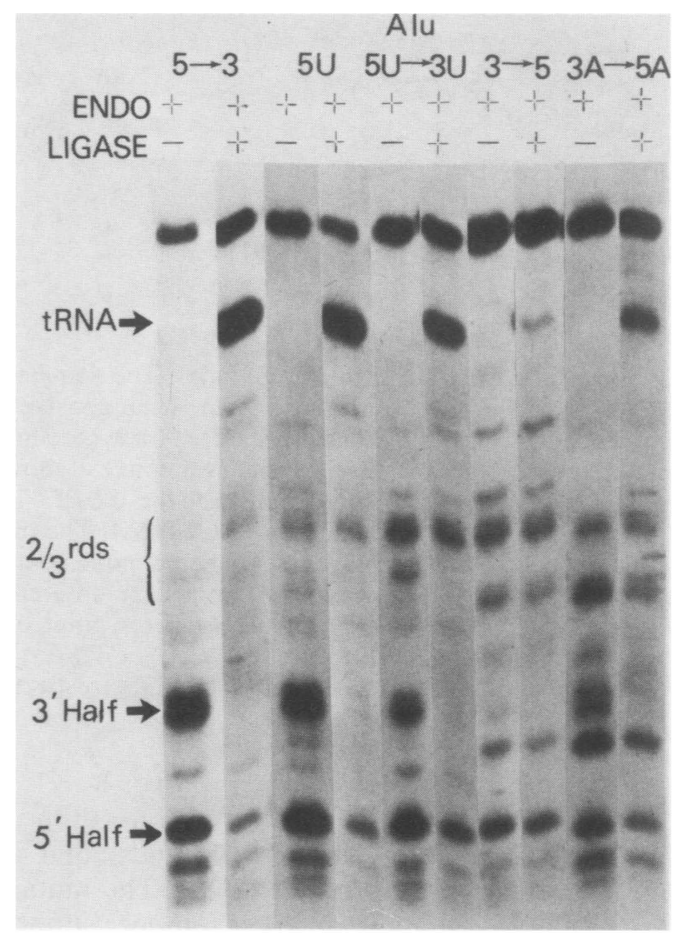

FIG. 3. In vitro splicing of Alu intron mutants. Assays were performed as described in Materials and Methods. All endprocessed pre-tRNAs were derived from a single transcription cocktail, and equal Cerekov counts were used in each cutting or splicing reaction mixture.

had wild-type residues at the $5^{\prime}$ and $3^{\prime}$ ends of its IVS, while the nonsplicers did not. Does the novel intron represent a totally inappropriate substrate, or are the splice junctionproximal sequences themselves inhibitory to splicing?

To address this question, $U$ residues were introduced in the Alu $5 \rightarrow 3$ intron at the $5^{\prime}$ and $3^{\prime}$ junctions (Alu5U $\rightarrow 3 \mathrm{U}$ ) or only at the $5^{\prime}$ junction (Alu5U). Additionally, A residues were substituted for the U's normally present at both intronexon junctions of Alu3 $\rightarrow 5$, creating Alu3A $\rightarrow 5 \mathrm{~A}$. The internal intron nucleotides were unchanged from those of the respective Alu parents (Fig. 1).

These three new mutants were transcribed in vitro in parallel with their Alu parents. All genes were transcribed and the primary transcripts were end-trimmed efficiently (data not shown). These end-processed transcripts were used in the in vitro splicing assay (Fig. 3).

The substitution of A residues at the splice junctions in the Alu3 $\rightarrow 5$ mutant had a clear effect on the splicing phenotype. The Alu3A $\rightarrow 5$ A RNA produced significant amounts of $3^{\prime}$ and $5^{\prime}$ half-molecules. Upon the addition of ligase, these half-molecules were converted to mature-size tRNA, in contrast to the products of Alu3 $\rightarrow 5$ RNA. However, both mutant RNAs generated some presumptive two-thirds and 5' half-molecules, suggesting that the junction alterations only partially rescue the splicing defect.

In contrast, the introduction of $U$ residues had significantly less effect on the ability of the Alu5 $\rightarrow 3$ precursor to be spliced. The Alu5U $\rightarrow 3 \mathrm{U}$ RNA produced both half-size and mature molecules. However, there was a substantial increase in the amount of two-thirds molecules generated from Alu5U $\rightarrow 3 \mathrm{U}$. Therefore, the endonucleolytic cleavage of the uridine-containing mutant appears to be less efficient. The 


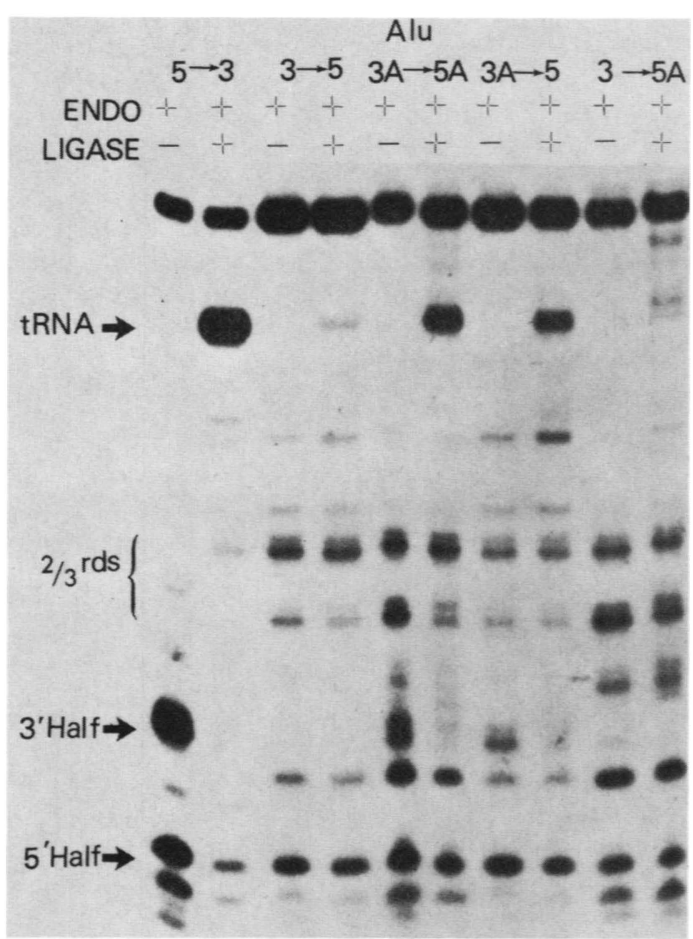

FIG. 4. In vitro splicing of Alu intron mutants altered only at the 3' splice junction. Assays were performed as described in Materials and Methods. All end-processed pre-tRNAs were derived from a single transcription cocktail, and equal Cerenkov counts were used in each cutting or splicing reaction mixture. introduction of a single $U$ residue at the $5^{\prime}$ splice junction (Alu5U) had virtually no effect on splicing.

Single base changes at the $3^{\prime}$ intron-exon junction affect splicing. The nonspliced mutants all exhibited partial, inefficient cutting at the 5 ' exon-intron junction. Furthermore, the splicing pattern observed for the mutants described above implied that changes proximal to the $3^{\prime}$ splice junction may more critically affect cleavage. To directly test this idea, single mutations were introduced in the intron nucleotide immediately at the $3^{\prime}$ junction.

A single A residue was introduced at the $3^{\prime}$ junction of the nonspliced Alu $3 \rightarrow 5$ mutant, creating Alu $3 \mathrm{~A} \rightarrow 5$. Conversely, a single $U$ residue replaced an $A$ at the $3^{\prime}$ junction of the spliced Alu3A $\rightarrow 5 \mathrm{~A}$, creating Alu3 $\rightarrow 5 \mathrm{~A}$ (Fig. 1). Each new mutant and its respective parent were transcribed in vitro. All mutant RNAs were efficiently synthesized and endprocessed (data not shown). The end-processed transcripts were used in the in vitro splicing assay shown in Fig. 4.

As previously shown, the Alu $3 \rightarrow 5$ mutant precursor generated predominantly 5 '-half and presumptive two-thirds molecules in the presence of endonuclease. However, the introduction of a single $A$ residue $(\mathrm{Alu} 3 \mathrm{~A} \rightarrow 5$ ) altered the cleavage products. Significant amounts of both $5^{\prime}$ and $3^{\prime}$ halves and mature-size tRNA were produced, but as evidenced by the appearance of two-thirds molecules, the cleavage was not totally efficient.

The Alu $3 \mathrm{~A} \rightarrow 5 \mathrm{~A}$ and Alu3 $\rightarrow 5 \mathrm{~A}$ mutant RNAs gave the converse result. The Alu $3 \mathrm{~A} \rightarrow 5 \mathrm{~A}$ precursor produced mature tRNA. However, the mutant which contained a $U$ residue at the $3^{\prime}$ junction did not; it exhibited inefficient cleavage only at the $5^{\prime}$ junction.

Identification of RNAs produced by in vitro splicing of Alu $5 \rightarrow 3$, Alu $3 \rightarrow 5$, and Alu3A $\rightarrow 5 A$ precursors by RNA fingerprinting. Two-dimensional separation of $\left[\alpha-{ }^{32}\right.$ P $]$ UTP-

TABLE 1. $\left[\alpha_{-}{ }^{32} \mathrm{P}\right]$ UTP-labeled $\mathrm{T}_{1}$ oligonucleotides of SUP53 intron mutants ${ }^{a}$

\begin{tabular}{|c|c|c|c|c|c|c|c|c|}
\hline $\begin{array}{c}\text { Oligo- } \\
\text { nucleotide } \\
\text { no. }\end{array}$ & Sequence $^{b}$ & Pre-tRNA & $\begin{array}{c}\text { Spliced } \\
\text { tRNA }\end{array}$ & $5^{\prime}$-half & $3^{\prime}$-half & IVS & $2 / 3 A^{d, e}$ & $2 / 3 B^{d, e}$ \\
\hline 1 & $\mathrm{Gp}(\mathrm{T} 1)$ & + & + & + & + & - & + & + \\
\hline 2 & UUGp (T13) & + & + & + & - & - & - & - \\
\hline 3 & UUUGp (T19) & + & + & + & - & - & - & - \\
\hline 4 & UCUAAGp (T17) & + & + & + & - & - & - & - \\
\hline 5 & CCUGp (T9) & + & + & + & - & - & - & - \\
\hline 6 & AUUCUAGp (T18) & + & - & + & - & - & - & _- \\
\hline 7 & AAAUAAUCUUG & $+^{c}$ & - & - & - & + & - & - \\
\hline $7 a$ & UAUUUCAAGp & $+^{d}$ & - & - & - & + & + & - \\
\hline $7 \mathrm{~b}$ & AAUUUCAAGp & $t^{e}$ & - & _ & _- & + & + & - \\
\hline 8 & AAAUACUCAGp & $+c$ & - & - & - & - & - & - \\
\hline $8 a$ & AUAUUUCUCAGp & $+^{d}$ & - & - & - & - & + & + \\
\hline $8 \mathrm{~b}$ & AUAUUACUCAGp & $+e$ & - & - & - & - & + & + \\
\hline 9 & CUCAGp (T12) & - & - & - & + & - & - & - \\
\hline 10 & UAUCGp (T16) & + & + & - & + & - & + & + \\
\hline 11 & AUGp (T10) & + & + & - & + & - & + & + \\
\hline 12 & AGp (T4) & + & + & - & + & - & + & + \\
\hline 13 & UUCGp (T14) & + & + & - & + & - & + & + \\
\hline 14 & AAUCUCUUAGp (T21) & + & + & - & + & - & + & + \\
\hline 15 & AUUCUA*CUCAGp (T22) & - & + & - & - & - & - & - \\
\hline
\end{tabular}

${ }^{a}\left[\alpha-{ }^{32} \mathrm{P}\right]$ UTP-labeled oligoribonucleotides derived from the pre-tRNAs and the in vitro splicing products of the Alu5 $\rightarrow 3$, Alu3 $\rightarrow 5$, and Alu3A $\rightarrow 5 \mathrm{~A}$ mutants are listed.

${ }^{b}$ Underlined sequence is the anticodon (CUA). The asterisk $\left(^{*}\right)$ in oligonucleotide 15 (splice junction) indicates the presence of a $2^{\prime}$-phosphate, which renders this junction insensitive to digestion by RNase $T_{1}$. The designations in parentheses refer to the tRNA ${ }_{3}^{\mathrm{Leu}}$ oglionucleotide nomenclature used by Peebles et al. (22, 23) and Greer et al. (7). Oligonucleotides 6 (T18) and 15 (T22) do not precisely correspond to their tRNA ber anticodon.

c Alu $5 \rightarrow 3$ mutant only.

${ }^{d}$ Alu $3 \rightarrow 5$ mutant only.

e Alu3A $\rightarrow$ 5A mutant only. 


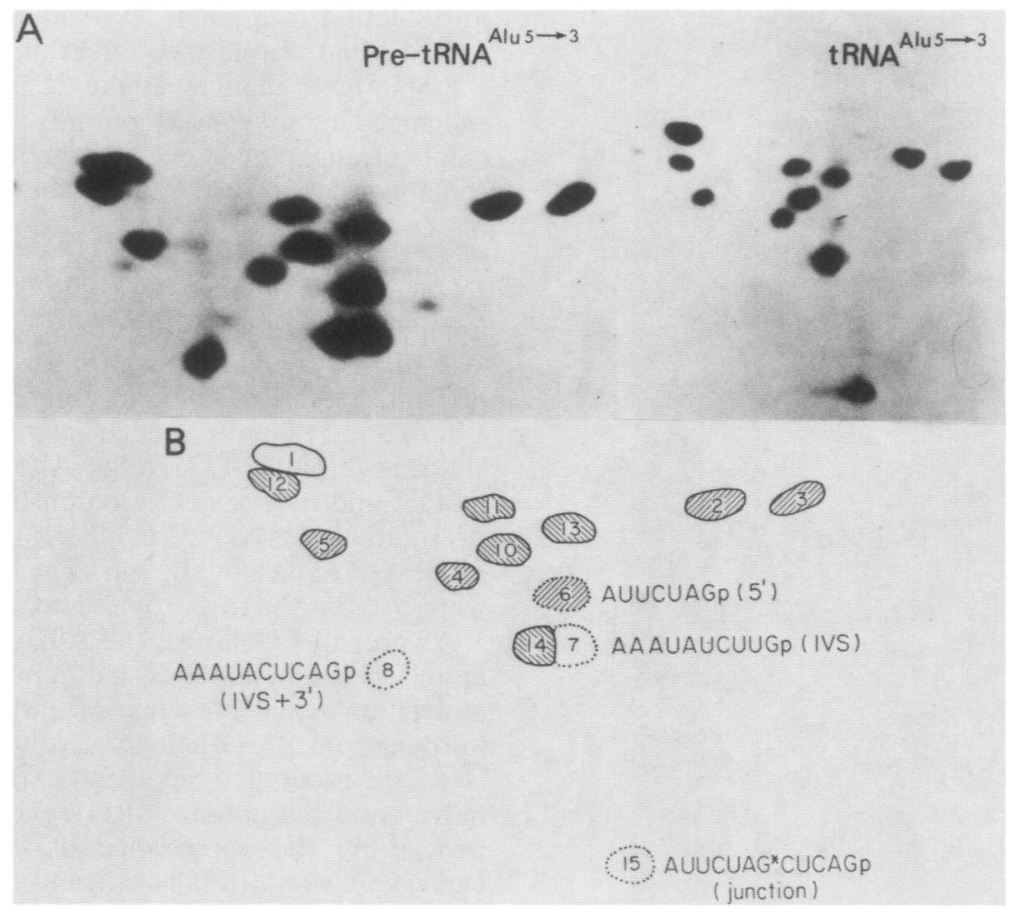

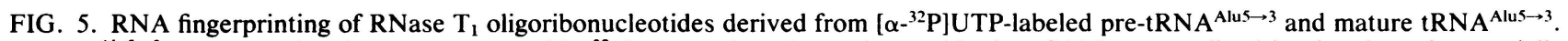
Pre-tRNA ${ }^{\text {Alus } \rightarrow 3}$, made in vitro in the presence of $\left[\alpha^{32}\right.$ P]UTP and $S$-adenosyl-L-methionine (SAM), was spliced in vitro by using partially purified tRNA endonuclease and ligase as described in Materials and Methods. The RNAs were digested with RNase $T_{1}$ as described in Materials and Methods. $T_{1}$ oligonucleotides were resolved by two-dimensional separation (RNA fingerprinting). (A) Fingerprints of pre-tRNA ${ }^{\text {Alus } \rightarrow 3}$ and mature tRNA ${ }^{\text {Alus } \rightarrow 3}$. First-dimension electrophoresis was left to right; second-dimension homochromatography was bottom to top. (B) Cartoon identifying spots. The sequence of each spot is listed in Table 1. Stippled spots were derived from the intron, the intron-exon junctions, or the exon-exon (splice) junction. The asterisk $\left(^{*}\right)$ in the splice junction oligonucleotide indicates the presence of a 2 '-phosphate, which renders the junction (G-P-pC) insensitive to RNase $T_{1}$. V/A, Oligonucleotides specific for the 5'-half tRNA molecule; aI, oligonucleotides specific for the $3^{\prime}$-half tRNA molecule.

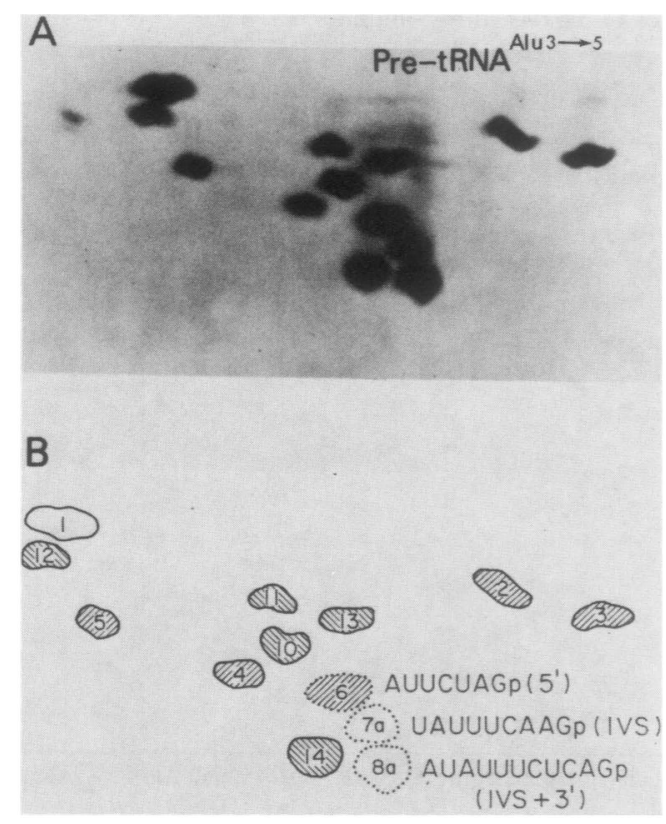

FIG. 6. RNA fingerprinting of RNase $T_{1}$ oligoribonucleotides derived from $\left[\alpha^{-32}\right.$ P]UTP-labeled pre-tRNA ${ }^{\text {Alu } 3 \rightarrow 5}$. Pre-tRNA ${ }^{\text {Alu } 3 \rightarrow 5}$, made in vitro in the presence of $\left[\alpha{ }^{-32}\right.$ P]UTP and SAM, was used as a substrate for partially purified tRNA endonuclease and ligase as described in Materials and Methods. The $T_{1}$ digestion products were separated as described in Material and Methods. (A) Fingerprint of labeled RNase $\mathrm{T}_{1}$ oligonucleotides was used to confirm the identity of RNAs produced by in vitro splicing (Fig. 5 through 8, Table 1). Since these RNAs are labeled with UTP alone, only a subset of the oligonucleotides for tRNA ${ }_{3}^{\text {Leu }}$ were seen $(7,22,23)$.

Figure 5 shows fingerprints of the Alu $5 \rightarrow 3$ precursor and mature RNA. Four oligonucleotides differed between the two sets of $T_{1}$ cleavage products. Through splicing of the precursor, three oligos, 6, 7, and 8, disappeared and a new oligo, 15, appeared in mature tRNA. Oligonucleotides 7 and 8 represented intron and intron- 3 '-half junction sequences, respectively. Oligo 6 contained the $3^{\prime}$ terminus of the $5^{\prime}$ half-molecule. In mature tRNA, oligo 6 and the mature portion of oligo 8 have been ligated to create oligo 15 . The $5^{\prime}$ half-molecule terminated in a $G$ residue (Fig. 1). In the ligated mature tRNA, no RNase $T_{1}$ cleavage occurred at this position due to a $2^{\prime}$-phosphate at the spliced junction $\left(\mathrm{G}_{-\mathrm{p}}^{-\mathrm{p}} \mathrm{p}\right)$. In vivo this phosphate is probably removed by an as yet unidentified phosphatase. However, with the partially purified splicing enzymes, little or no phosphatase activity remains (7). Therefore, this junction is refractory to $T_{1}$ cleavage.

The endonuclease cleavage products of pre-tRNA $A^{\text {Alu3 } \rightarrow 5}$

pre-tRNA ${ }^{\mathrm{Alu} 3 \rightarrow 5}$. First-dimension electrophoresis was left to right; second-dimension homochromatography was bottom to top. (B) Cartoon identifying spots. The sequence of each spot is listed in Table 1. Stippled spots, Intron or intron-exon junction; was, $5^{\prime}$-half tRNA molecules; $\mathrm{ND}, 3^{\prime}$-half tRNA molecules. 


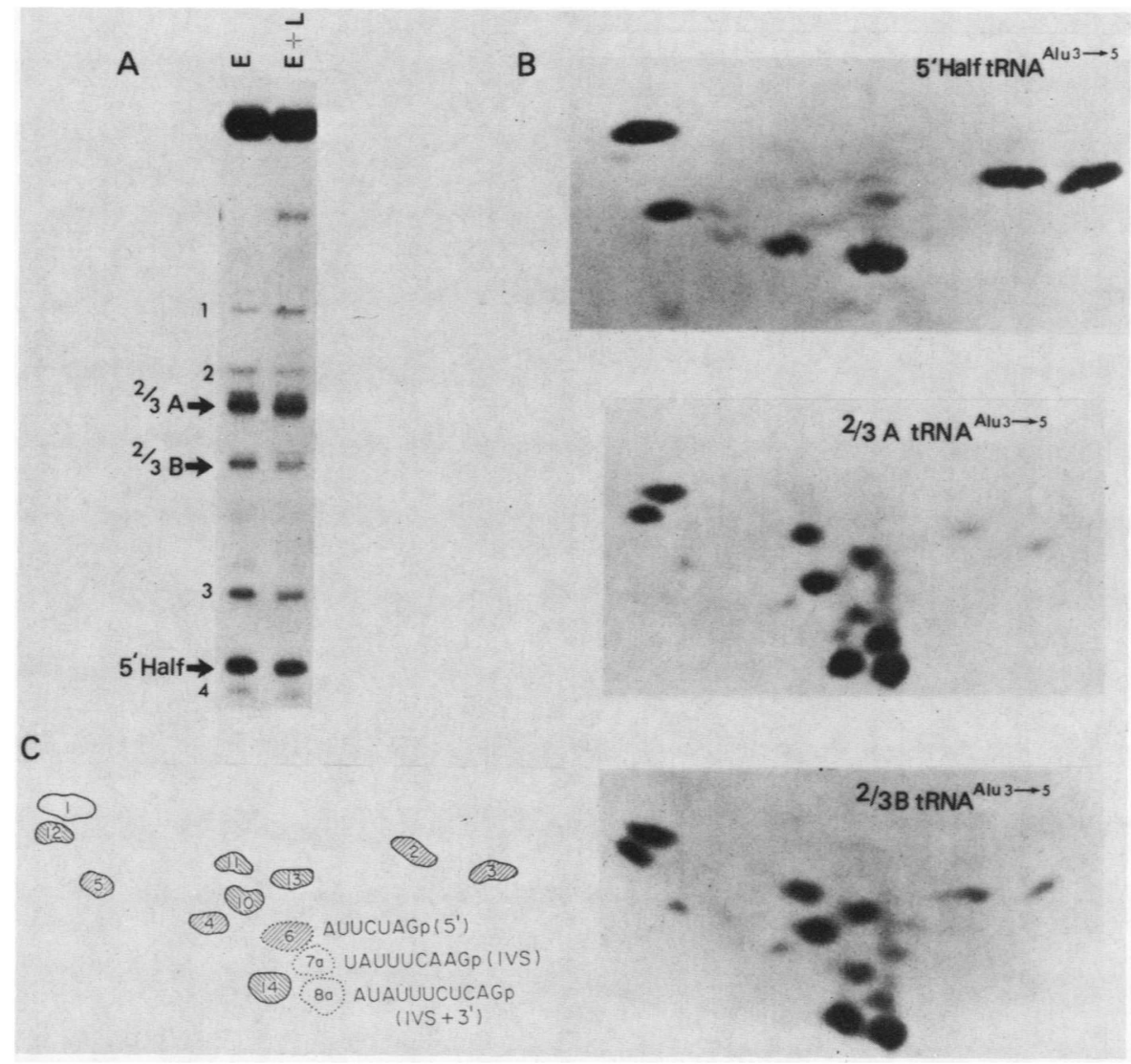

FIG. 7. RNA fingerprinting of RNase $T_{1}$ oligoribonucleotides derived from the in vitro splicing products of [ $\left.\alpha-{ }^{32} \mathrm{P}\right] \mathrm{UTP}-\mathrm{labeled}$ pre-tRNA ${ }^{\text {Alu } 3 \rightarrow 5}$. Pre-tRNA ${ }^{\text {Alu } 3 \rightarrow 5}$, made in vitro in the presence of $\left[\alpha^{-32}\right.$ P $]$ UTP and SAM, was analyzed in vitro by using partially purified tRNA endonuclease and ligase as described in Materials and Methods. RNAs were digested with RNase $T_{1}$. The $T_{1}$ digestion products were resolved as described in Materials and Methods. (A) Splicing gel of pre-tRNA Alu3 $\rightarrow 5.2 / 3 \mathrm{~A}, 2 / 3 \mathrm{~B}$, and $5^{\prime}$-half RNAs were identified initially by size. Identity of numbered bands ( 1 through 4 ) is discussed in the text. E, Endonuclease alone; E + L, endonuclease plus ligase. (B) RNA fingerprinting of $2 / 3 \mathrm{~A}, 2 / 3 \mathrm{~B}$, and 5'-half RNAs. First-dimension electrophoresis was left to right; second-dimension homochromatography was bottom to top. (C) Cartoon identifying spots. The sequence of each spot is listed in Table 1. Stippled spots, Intron or intron-exon junction; ezs, 5'-half molecule; $\mathbf{N}, 3^{\prime}$-half molecule.

are shown in Fig. 6 and 7. Comparing the RNase $T_{1}$ pattern generated from the Alu $5 \rightarrow 3$ and Alu $3 \rightarrow 5$ precursor RNAs, two changes were seen (Fig. 5 and 6). The migration of the intron (oligos 7 and 7a) and intron plus $3^{\prime}$-half (oligos 8 and 8a) molecules was altered, reflecting their different size and sequence (Table 1). The mature-domain oligonucleotides for the two RNAs were identical.

Figure 7 shows a typical splicing assay with Alu3 $\rightarrow 5$ RNA and the fingerprints of the labeled bands. The 5'-half tRNA band showed the expected oligonucleotides for genuine $5^{\prime}$ half-molecules generated by endonuclease. Band two-thirds A had a pattern consistent with its generation by cleavage only at the $5^{\prime}$ exon-intron jucntion, containing oligos indicative of the total intron (oligo 7a), intron- 3 '-half junction (oligo 8a), and $3^{\prime}$ half (oligos 10 through 14). Similarly, mature $3^{\prime}$ half oligos were in the two-thirds $B$ fingerprint. However, only molar amounts of the $3^{\prime}$ intron-exon junction (oligo 8a) were seen. The intron-specific oligo 7a was present in only trace amounts. The implies that the two-thirds B molecule was generated either by cleavage within the intron or by degradation.

The bands numbered 1 through 4 in Fig. 7A were also analyzed. While their identity has not been precisely determined, they exhibited a pattern consistent with molecules nicked in the $3^{\prime}$ half (bands 1 and 2) and partial $5^{\prime}$ halfmolecules (bands 3 and 4). The pattern of these aberrant bands was generally consistent for a given mutant and is probably a function of other endonuclease activities present in the partially purified endonuclease and ligase preparations used.

Figure 8 illustrates the fingerprints of the Alu3A $\rightarrow 5 \mathrm{~A}$ precursor and mature tRNA. As described above, after splicing the intron and intron-exon junction olignucleotides, $6,7 \mathrm{~b}$, and $8 \mathrm{~b}$, disappeared and the splice junction oligo, 15 , appeared. Again, this splice junction oligo was not cleaved by RNase $T_{1}$ due to the presence of a $2^{\prime}$-phosphate $\left(G^{-p} p C\right)$. Therefore, this precursor produced properly spliced $S U P 53$ tRNA. The results of fingerprint analysis of the two-thirds molecules generated by cleavage of Alu3A $\rightarrow 5 \mathrm{~A}$ RNA were in accord with the pattern elucidated for similar molecules derived from $3 \rightarrow 5$ cleavage (see Fig. 7).

Suppressor function of Alu and Stu intron mutants in vivo. The altered genes were tested for their ability to produce functional suppressor tRNA in vivo upon integration into the S. cerevisiae genome (Table 2). In all cases, the suppressor

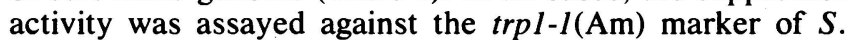
cerevisiae SL183-21C. Cells were transformed with a linear DNA fragment containing the SUP53 allele and the LEU2 
marker. $\mathrm{Leu}^{+}$transformants were secondarily screened for their ability to grow at different cell densities on minimal medium minus tryptophan. The more efficiently the tRNA suppresses the amber codon, the lower the cell density at which growth is observed. The parent strain (SL183-21C) exhibited no growth at any density tested.

The RNA processing and suppression phenotypes of $S U P 53$, Alu5 $\rightarrow 3, \Delta$ IVS, and INS mutants have been described previously (29). Briefly, the down-suppressor phenotype of $\Delta$ IVS and Alu $5 \rightarrow 3$ is correlated with the lack of an anticodon wobble base modification ( $\mathrm{C}$ to 5 -methyl-C). Only the totally intact SUP53 pre-tRNA was methylated in this position. Since none of these intron mutants contained the anticodon modification (data not shown), the highest level of suppression expected is that for $\Delta$ IVS or Alu5 $\rightarrow 3$. Therefore, the level of suppression seen with these additional mutants presumably reflects the extent of other RNA processing steps, notably splicing.

The in vitro-nonspliced pre-tRNAs (Alu3 $\rightarrow 5$, Alu3 $\rightarrow 5 \mathrm{~A}$, Stu $5 \rightarrow 3$, and Stu3 $\rightarrow 5$ ) were null suppressors. The three mutants, which exhibited inefficient splicing in vitro, showed decreased suppressor function in vivo (Alu $5 \mathrm{U} \rightarrow 3 \mathrm{U}$, Alu $3 \mathrm{~A} \rightarrow 5 \mathrm{~A}$, and Alu3A $\rightarrow 5$ ). Furthermore, their level of suppressor function was consistent with the amount of mature tRNA produced in vitro. Alu $5 \mathrm{U} \rightarrow 3 \mathrm{U}$ precursor was spliced and suppressed more efficiently than Alu3 $\rightarrow$ $\rightarrow$ A and Alu $3 \mathrm{~A} \rightarrow 5$, which produced progressively less mature tRNA (Fig. 3 and 4).

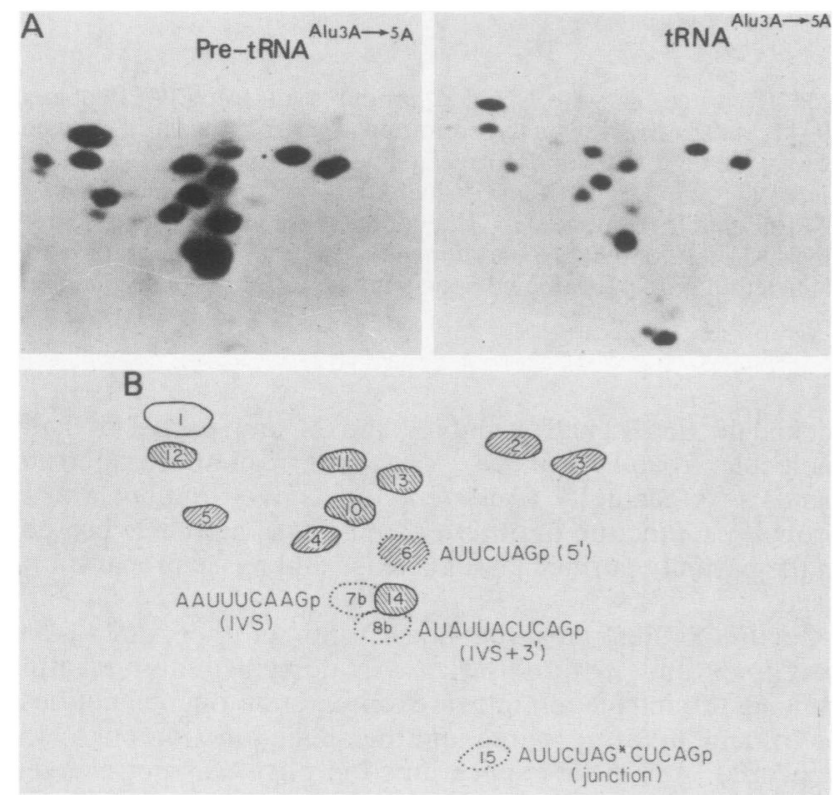

FIG. 8. RNA fingerprinting of RNase $T_{1}$ oligoribonucleotides derived from $\left[\alpha-{ }^{32} \mathrm{P}\right] U T \mathrm{UP}$-labeled pre-tRNA Alu3A $\rightarrow 5 \mathrm{~A}$ and mature tRNA $A^{\mathrm{Alu} 3 \mathrm{~A} \rightarrow 5 \mathrm{~A}}$. Pre-tRNA $\mathrm{Alu}^{\mathrm{A} A \rightarrow 5 \mathrm{~A}}$, made in vitro in the presence of $\left[\alpha{ }^{32}\right.$ P]UTP and SAM, was spliced by using partially purified tRNA endonuclease and ligase as described in Materials and Methods. The $\mathrm{T}_{1}$ digestion products were resolved as described. (A) RNA fingerprinting of pre-tRNA $A^{\text {Alu } 3 A \rightarrow 5 A}$ and mature tRNA Alu3A $\rightarrow 5 A$. Firstdimension electrophoresis was left to right; second-dimension homochromatography was bottom to top. (B) Cartoon identifying spots. The sequence of each spot is listed in Table 1. Stippled spots, Intron exon, or exon-exon (splice) junction; 10,5 'half molecule; $\Delta \backslash, 3^{\prime}$-half molecule. Asterisk $\left({ }^{*}\right)$ in the splice junction oligonucleotide indicates the presence of a $2^{\prime}$-phosphate which renders the junction (G-P-pC) insensitive to RNase $T_{1}$.
TABLE 2. Suppressor function of Alu and Stu intron mutants in vivo $^{a}$

\begin{tabular}{llc}
\hline \multicolumn{1}{c}{ tRNA } & \multicolumn{1}{c}{ Splicing $^{b}$} & Suppression $^{c}$ \\
\hline SUP53 & Yes & +++++ \\
SIVS & $\bar{C}^{d}$ & +++ \\
Alu5 $\rightarrow 3$ & Yes & +++ \\
Alu5U $\rightarrow$ 3U & Yes (decreased efficiency) & ++ \\
Alu3 $\rightarrow 5$ & No (2/3) & - \\
Alu3A $\rightarrow 5 \mathrm{~A}$ & Yes (decreased efficiency) & + \\
Alu3A $\rightarrow 5$ & Yes (decreased efficiency) & $+/-$ \\
Alu3 $\rightarrow 5 \mathrm{~A}$ & No (2/3) & - \\
INS & No & - \\
Stu5 $\rightarrow 3$ & No (2/3) & - \\
Stu3 $\rightarrow 5$ & No (2/3) & - \\
\hline
\end{tabular}

${ }^{a}$ Suppressor function of SUP53 and Alu and Stu intron mutants was quantified as described in Materials and Methods. S. cerevisiae SL183-21C was transformed with a linear restriction fragment containing both the tRNA gene and the $L E U 2$ marker. Serial dilutions of cultures of $\mathrm{Leu}^{+}$transformants were plated on minimal medium minus tryptophan to test their ability to

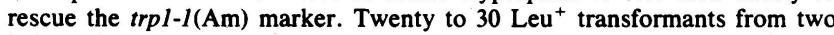
independent yeast transformations with each tRNA gene were tested. Plates were scored for growth after 3 to 4 days at $30^{\circ} \mathrm{C}$.

${ }^{b}$ As discussed in the text, decreased efficiency of splicing refers to the production of both mature tRNA and two-thirds (2/3) molecules. $2 / 3$ indicates that the precursor evidences only inefficient cleavage at the 5 ' splice junction and no significant amount of mature tRNA.

c Strong suppressor $(+++++)$, growth at low cell density $\left(10^{1}\right.$ cells); weaker suppressor $(+++,++$, and +$)$, growth only at a higher cell density $\left(10^{3}, 10^{4}\right.$, and $10^{5}$, respectively); extremely weak suppressor $(+/-)$, marginal growth; null suppressor $(-)$, no growth at $10^{5}$ cells. SL183-21C showed no growth in the absence of tryptophan at any cell density.

${ }^{d} \_, \Delta$ IVS contains no intron.

\section{DISCUSSION}

Intron mutations can have a profound effect on the processing of a precursor into mature tRNA both in vitro and in vivo. Each mutation was initially assayed by its ability to serve as a splicing substrate in vitro. However, the in vivo function of each tRNA faithfully reflected its splicing phenotype.

Unlike the numerous tRNA mutants that exhibit RNAprocessing defects $(11-13,18,21,35)$, these intron changes did not alter the mature portion of the precursor. Rather, at this level of detection, these mutant pre-tRNAs were efficiently end-processed. As previously noted (29), the activity of the end-maturation enzymes is a sensitive indicator of appropriate mature-domain structure. Rather, the splicing pattern of these mutants was affected by changes only in the intron itself. Single-nucleotide changes can have a drastic effect on the predicted secondary structure of the intron (Fig. 9). Additionally, while tRNA precursors exhibit no sequence conservation at exon-intron junctions or within introns (20), certain primary sequences at these junctions may be inappropriate.

These data imply that the most sensitive step in tRNA splicing is endonucleolytic cleavage. As seen in Fig. 2 through 4, all the appropriate half-molecules were ligated; in no case was the ligation step obviously inhibited. Rather, all poorly spliced or unspliced precursors were blocked in the production of $3^{\prime}$ half-molecules. With every defective splicing mutant, the products of inefficient cleavage at the $5^{\prime}$ splice junction were seen $\left(5^{\prime}\right.$ half-molecules and two-thirds molecules). The products of cleavage solely at the $3^{\prime}$ splice junction were never detected.

While the mechanism of tRNA half-molecule ligation in $S$. cerevisiae is well characterized $(7,23 \mathrm{a})$, similar information about the endonuclease is limited (22). Furthermore, little is 


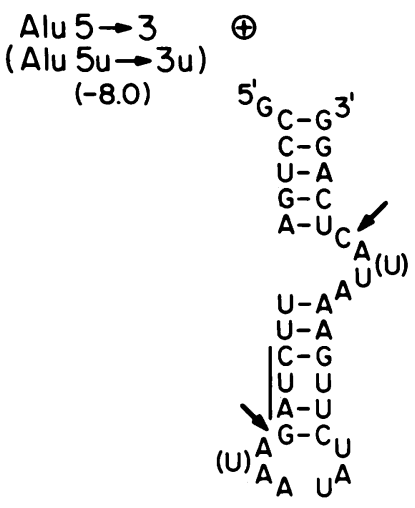

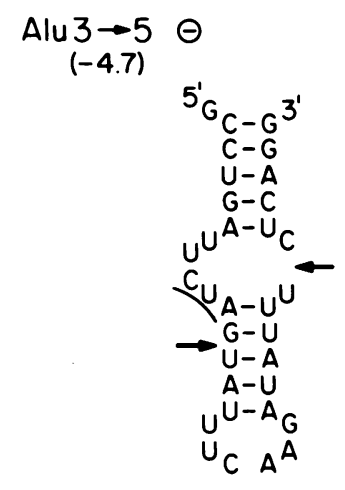

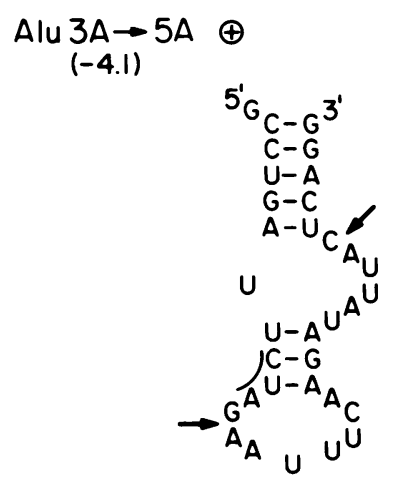

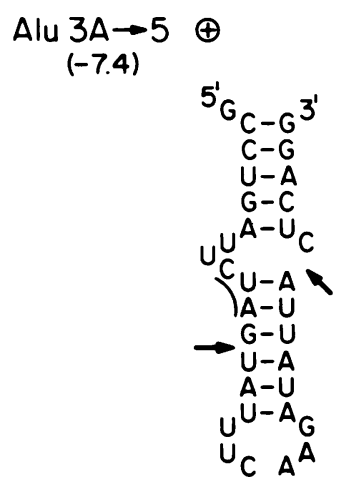

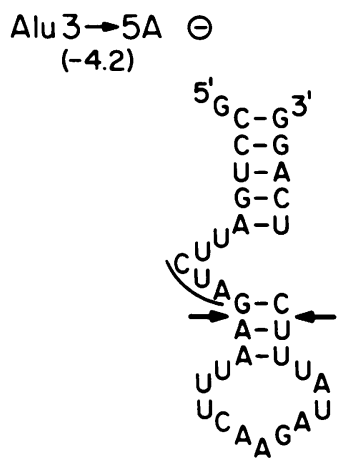

FIG. 9. Computer-generated secondary structures of Alu family mutants. The secondary structures of Alu family mutant precursor tRNAs anticodon stem-intron region were determined with the RNA secondary structure program of Zuker provided by the BIONET National Computer Resource for Molecular Biology. + and - , Splicing phenotype of each mutant. Numbers in parentheses indicate the free energy calculated for the structure shown. The anticodon (CUA) is indicated in each structure; arrows indicate known or predicted splice junctions.

known about the interaction(s) between these enzymes and their substrate. Therefore, the important features for endonuclease-substrate recognition and cleavage has been inferred from the common features of the substrates.

By analogy to other tRNA maturation enzymes, endonuclease must recognize the structure of the mature domain of the precursor, which retains the conserved conformation determined for mature tRNA ${ }^{\text {Phe }}$ (10). For instance, endonuclease activity is inhibited by mature tRNA (P. Green and J. Abelson, unpublished observation). The mutations which prevent splicing in vivo map to the $\mathrm{D}$ stem-loop, the variable stem-loop, and the anticodon stem $(2,16,18)$. Therefore, the conserved position of the intron and the constant length of the anticodon stem in precursor tRNAs $(19,20)$ have been deemed important for correct endonuclease cleavage. Neither feature was affected by these mutations, nor was the mature domain altered.

Additionally, the $S$. cerevisiae pre-tRNA introns have a common structure $(14,19,20,31)$. Each intron folds to form an extension of the anticodon stem. The splice junctions are generally in single-stranded loops and are a constant distance apart (6 base pairs [9]). This extended anticodon stem is stabilized by base-pairing between the anticodon and the intron, allowing the stem residues to be highly base-stacked while the loop nucleotides are not. Previously, we showed that disruption of anticodon-intron complementarity was not sufficient per se to inhibit splicing of a precursor tRNA (29). Otherwise, this precursor conforms to the expected structure. In contrast, two $S$. pombe intron-containing precursors were inefficiently spliced in $S$. cerevisiae extracts. It has been suggested these pre-tRNAs do not form a stable extended anticodon stem $(6,30)$.

The two intron families $(5 \rightarrow 3$ and $3 \rightarrow 5)$ presented here retained the potential to form such a stable extended stem. However, the secondary structures of these mutants predict that only a subset actually form the structure. Alu5 $\rightarrow 3$, Alu5U, and Alu5U $\rightarrow 3 \mathrm{U}$ pre-tRNAs conformed to the expected structure (Fig. 9), and these precursors were readily spliced (Fig. 2 and 3). In contrast, two unspliced precursors (Alu3 $\rightarrow 5$ and Alu3 $\rightarrow 5 \mathrm{~A}$ ) exhibited severe distortion of the extended stem. These precursors were predicted to form a large, single-stranded loop $5^{\prime}$ of the intron. Formation of this loop places the $5^{\prime}$ splice junction in a double-stranded region. However, these mutant RNAs were inefficiently cleaved at the $5^{\prime}$ junction, suggesting that its conformation is less critical. For instance, the most stable structure for $S$.

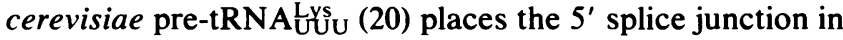
a double-stranded region.

Perhaps a more critical consequence of the predicted stem distortion is its effect on the $3^{\prime}$ splice junction. The precursors which produced mature tRNA (Alu5 $\rightarrow 3$, Alu5U, Alu5U $\rightarrow 3 \mathrm{U}$, and Alu3A $\rightarrow 5 \mathrm{~A}$ ) had substantial singlestranded regions at the $3^{\prime}$ splice junction; loops were consistent with those observed among the natural $S$. cerevisiae precursors (20). Furthermore, the distance between the two splice junctions was consistent with that predicted for the natural pre-tRNAs (9). In contrast, the unspliced mutant pre-tRNAs (Alu3 $\rightarrow 5$ and Alu3 $\rightarrow 5 \mathrm{~A}$ ) exhibited severe alter- 
ation of the $3^{\prime}$ junction. In the extreme case, Alu3 $\rightarrow 5 \mathrm{~A}$ lacked any single-stranded region at this junction, and the two splice junctions were virtually apposed. Pre-tRNA ${ }^{\text {Alu } 3 \rightarrow 5}$ formed an unusually small junction loop, and the distance between the junctions was significantly smaller. The smallest 3' loop size predicted for an $S$. cerevisiae pre-tRNA is three nucleotides (20). An unspliced mutant of pre-tRNA ${ }_{3}^{\text {Leu }}$ has only a 2-nucleotide $3^{\prime}$ junction loop (25). Furthermore, by precise deletion at the $3^{\prime}$ splice junction of pre-tRNA ${ }^{\text {Pro }}$, Knapp and co-workers have shown that 2 nucleotides is the minimum loop size for total endonuclease cleavage (G. Knapp, personal communication).

One apparent exception among the mutated precursors was Alu3A $\rightarrow 5$. This spliced RNA contained only 1 nonbase-paired nucleotide at the $3^{\prime}$ junction. The distance between the splice junctions was not significantly different from that predicted for its unspliced parent. While this precursor did form a very stable extended anticodon stem, it was very inefficiently spliced both in vitro and in vivo.

Since knowledge about the mechanism of endonuclease cleavage is limited, the precise order of cleavage events, or whether there is an obligate order, is unknown. Peebles et al. (22) noted the appearance of presumptive two-thirds molecules in vitro from a natural pre-tRNA. Analysis of these molecules showed that they were derived by a single cleavage at the 3' splice junction (P. Hellung-Larsen, C. L. Peebles, and J. Abelson, unpublished observations; cited in reference 23 ). In contrast, the two-thirds molecules examined in this study were generated by a single cut at the $5^{\prime}$ junction. Whether either of these represents true reaction intermediates cannot be elucidated at this time. However, the two-thirds molecules derived from mutant precursors emphasize that cleavage at the $5^{\prime}$ junction must be less affected by intron alterations.

A final point to consider in a structural analysis of these intron-mutated precursor tRNAs is the effect of ligands which are known to interact with a tRNA molecule. Polyamines and magnesium ions are critical for the maintenance of mature tRNA tertiary structure $(10,24)$. The pattern of precursor residues sensitive to structure-probing nucleases differs when magnesium or spermidine is present (14). Peebles et al. (22) found that spermidine is necessary for correct cleavage by tRNA endonuclease. Both $\mathbf{M g}^{2+}$ and spermidine were present in all the cleavage-ligation assays presented here. However, no change in the cleavage pattern of Alu $5 \rightarrow 3$ or Alu3 $\rightarrow 5$ precursor was observed after the magnesium or spermidine concentration was varied over a broad range (data not shown).

These results are consistent with the idea that correct, efficient splicing is dependent on the intron's forming a stable extension of the anticodon stem and retaining the $3^{\prime}$ splice junction in a single-stranded region. However, the Alu5U $\rightarrow 3 \mathrm{U}$ and Stu $5 \rightarrow 3$ mutant precursors suggest that the primary sequence at the $3^{\prime}$ splice junction may be important per se. The predicted secondary structures for these mutants conform to that exemplified by the readily spliced Alu $5 \rightarrow 3$ precursor. However, the Stu mutant was cleaved only at the $5^{\prime}$ junction, and the Alu5U $\rightarrow 3 \mathrm{U}$ was spliced less efficiently (Fig. 2 and 3). While the splice junction sequences of the Alu mutant are not unique, the G-G $5^{\prime}$ and C-C $3^{\prime}$ Stu mutant junctions are not found among the natural pre-tRNAs (20). Raymond and Johnson (25) have reported that a mutant with a similar $3^{\prime}$ splice junction was refractory to the splicing enzymes. Therefore, these data suggest that the splice junction-proximal primary sequence may prove to be critical for endonuclease recognition and cleavage.

\section{ACKNOWLEDGMENTS}

We thank Eric Phizicky, Chris Greer, Andy Newman, and Rich Schwartz for their input and insights during the course of this work and Gayle Knapp for many stimulating discussions. Special thanks to Linda Brubaker, Connie Katz, and Donna Walker for preparation of the manuscript.

Computer resources were provided by BIONET National Computer Research for Molecular Biology, whose funding is provided by the Biomedical Research Technology Program, Division of Research Resources, National Institutes of Health (grant 1-U41-RR01685-02). This work was supported by the American Cancer Society (grant ACS NP302D) and by Public Health Service grant GM-32637 from the National Institutes of Health to J.A. M.C.S. was supported by a Public Health Service Genetics Training Grant (Biology Department, University of California, San Diego).

\section{LITERATURE CITED}

1. Andreadis, A., Y.-P. Hsu, G. B. Kohlhaw, and P. Schimmel. 1982. Nucleotide sequence of yeast LEU2 shows 5 ' non-coding region has sequences cognate to leucine. Cell 31:319-325.

2. Baldi, M. I., E. Mattocia, and G. P. Tocchini-Valentini. 1983. Role of RNA structure in splicing: excision of the intervening sequence in yeast $\mathrm{tRNA}^{\mathrm{Leu}}{ }_{3}$ is dependent on the formation of a D stem. Cell 35:109-115.

3. Colby, D., P. S. Leboy, and C. Guthrie. 1981. Yeast tRNA precursor mutated at a splice junction is correctly processed in vivo. Proc. Natl. Acad. Sci. USA 78:415-419.

4. Engelke, D. R., P. Gegenheimer, and J. Abelson. 1985. Nucleolytic processing of a tRNA ${ }^{\text {Arg }}$-tRNA ${ }^{\text {Asp }}$ dimeric precursor by a homologous component from Saccharomyces cerevisiae. J. Biol. Chem. 260:1271-1279.

5. Fischoff, D. A., R. H. Waterston, and M. V. Olson. 1984. The yeast cloning vector $\mathrm{YEp} 13$ contains a $\mathrm{RRNA}^{\mathrm{Leu}}{ }_{3}$ gene that can mutate to an amber suppressor. Gene 27:239-251.

6. Gamulin, V., J. Mao, B. Appel, M. Sumner-Smith, F. Yamao, and D. Soll. 1983. Six Schizosaccharomyces pombe tRNA genes including a gene for a tRNA ${ }^{\mathrm{Lys}}$ with an intervening sequence which cannot base pair with the anticodon. Nucleic Acids Res. 11:8537-8546.

7. Greer, C. L., C. L. Peebles, P. Gegenheimer, and J. Abelson. 1983. Mechanism of action of a yeast RNA ligase in tRNA splicing. Cell 32:537-546.

8. Johnson, J. D., R. Ogden, P. Johnson, J. Abelson, P. Dembeck, and K. Itakura. 1980. Transcription and processing of a yeast tRNA gene containing a modified intervening sequence. Proc. Natl. Acad. Sci. USA 77:2564-2568.

9. Kang, H. S., R. C. Ogden, G. Knapp, C. L. Peebles, and J. Abelson. 1979. Structure of yeast tRNA precursors containing intervening sequences, p. 69-84. In R. Axel. T. Maniatis, and F. Fox (ed.), Eukaryotic gene regulation. Academic Press, Inc., New York.

10. Kim, S.-H. 1979. Crystal structure of yeast tRNA ${ }^{\text {Phe }}$ and general structural features of other tRNAs, p. 83-100. In P. R. Schimmel, D. Soll, and J. N. Abelson (ed.), Transfer RNA: structure, properties and recognition. Cold Spring Harbor Laboratory, Cold Spring Harbor, N.Y.

11. Koski, R. A., S. G. Clarkson, J. Kurjan, B. D. Hall, and M. Smith. 1980. Mutations of the yeast SUP4 tRNA ${ }^{\text {Tyr }}$ locus: transcription of the mutant genes in vitro. Cell 22:415-425.

12. Koski, R. A., M. Worthington, D. S. Allison, and B. D. Hall. 1982. An in vitro RNA polymerase III system from $S$. cerevisiae: effects of deletions and point mutations upon SUP4 gene transcription. Nucleic Acids Res. 10:8127-8143.

13. Kurjan, J., B. D. Hall, S. Gillam, and M. Smith. 1980. Mutations at the yeast SUP4 tRNA ${ }^{\text {Tyr }}$ locus: DNA sequence changes in mutants lacking suppressor activity. Cell 20:701-709.

14. Lee, M.-C., and G. Knapp. 1985. Transfer RNA splicing in Saccharomyces cerevisiae: secondary and tertiary structures of the substrates. J. Biol. Chem. 260:3108-3115.

15. Liebman, S. W., Z. Srodulski, C. R. Reed, J. W. Stewart, F. Sherman, and G. Brennan. 1984. Yeast amber suppressors corresponding to tRNA ${ }^{\mathrm{Leu}}{ }_{3}$ genes. J. Mol. Biol. 178:209-226.

16. Mattocia, E., M. I. Baldi, G. Pande, R. Ogden, and G. Tocchini- 
Valentini. 1983. Mutation in the A block of the yeast tRNA ${ }^{\text {Leu }}{ }_{3}$ gene that allows transcription but abolishes splicing and $5^{\prime}$ end maturation. Cell 32:67-76.

17. Newman, A. J., R. C. Ogden, and J. Abelson. 1983. tRNA gene transcription in yeast: effects of specified base substitutions in the intragenic promoter. Cell 35:117-125.

18. Nishikura, K., J. Kurjan, B. D. Hall, and E. M. DeRobertis. 1982. Genetic analysis of the processing of a spliced tRNA. EMBO J. 1:263-268.

19. Ogden, R. C., G. Knapp, C. L. Peebles, H. S. Kang, J. S. Beckmann, P. F. Johnson, S. A. Fuhrman, and J. N. Abelson. 1980. Enzymatic removal of intervening sequences in the synthesis of yeast tRNA, p. 173-190. In D. Soll, J. N. Abelson, and P. R. Schimmel (ed.), Transfer RNA: biological aspects. Cold Spring Harbor Laboratory, Cold Spring Harbor, N.Y.

20. Ogden, R. C., M.-C. Lee, and G. Knapp. 1984. Transfer RNA splicing in Saccharomyces cerevisiae: defining the substrates. Nucleic Acids Res. 12:9367-9382.

21. Pearson, D., I. Willis, H. Hottinger, J. Bell, A. Kumar, U. Leupold, and D. Soll. 1985. Mutations preventing expression of sup3 tRNA ${ }^{\text {Ser }}$ nonsense suppressors of Schizosaccharomyces pombe. Mol. Cell. Biol. 5:808-815.

22. Peebles, C. L., P. Gegenheimer, and J. Abelson. 1983. Precise excision of intervening sequences from precursor tRNAs by a membrane-associated yeast endonuclease. Cell 32:525-536.

23. Peebles, C. L., R. C. Ogden, G. Knapp, and J. Abelson. 1979. Splicing of yeast tRNA precursors: a two-stage rection. Cell 18:27-35.

23a.Phizicky, E., R. Schwartz, and J. Abelson. 1986. S. cerevisiae tRNA ligase: purification of the protein and isolation of the structural gene. J. Biol. Chem. 261:2978-2986.

24. Quigley, G. J., M. M. Teeter, and A. R. Rich. 1978. Structural analysis of spermine and magnesium ion binding to yeast phenylalanine transfer RNA. Proc. Natl. Acad. Sci. USA 75: 64-68.

25. Raymond, G., and J. D. Johnson. 1983. The role of non-coding
DNA sequences in transcription and processing of a yeast tRNA. Nucleic Acids Res. 11:5969-5988.

26. Rothstein, R. J. 1983. One-step gene disruption in yeast. Methods Enzymol. 101:202-212.

27. Shaw, K. J., and M. V. Olson. 1984. Effects of altered 5'flanking sequences on the in vivo expression of a Saccharomyces cerevisiae tRNA ${ }^{\mathrm{Tyr}}$ gene. Mol. Cell. Biol. 4:657-665.

28. Southern, E. M. 1974. An improved method for transferring nucleotides from electrophoresis strips in thin layers of ionexchange cellulose. Anal. Biochem. 62:317-318.

29. Strobel, M. C., and J. Abelson. 1986. Effect of intron mutations on processing and function of Saccharomyces cerevisiae SUP53 tRNA in vitro and in vivo. Mol. Cell. Biol. 6:2663-2673.

30. Sumner-Smith, M., H. Hottinger, I. Willis, T. L. Koch, R. Arentzen, and D. Soll. 1984. The sup8 tRNA ${ }^{\text {Leu }}$ gene of Schizosaccharomyces pombe has an unual intervening sequence and reduced pairing in the anticodon stem. Mol. Gen. Genet. 197:447-452.

31. Swerdlow, H., and C. Guthrie. 1984. Structures of introncontaining tRNA precursors: analysis of solution conformation using chemical and enzymatic probes. J. Biol. Chem. 259: 5197-5207.

32. Volckaert, G., and W. Fiers. 1977. A micromethod for base analysis of ${ }^{32} \mathrm{P}$-labelled oligonucleotides. Anal. Biochem. 83:222-227.

33. Volckaert, G., and W. Fiers. 1977. Micro thin layer techniques for rapid sequence analysis of ${ }^{32} \mathrm{P}$-labelled RNA: double digestion and pancreatic RNase analysis. Anal. Biochem. 83: 228-239.

34. Volckaert, G., W. Min Jou, and W. Fliers. 1976. Analysis of ${ }^{32}$ P-labelled bacteriophage MS2 RNA by a minifingerprint procedure. Anal. Biochem. 72:433-446.

35. Willis, I., H. Hottinger, D. Pearson, V. Chisholm, U. Leupold, and D. Soll. 1984. Mutations affecting excision of the intron from a eukaryotic dimeric tRNA precursor. EMBO J. 3:15731580 . 